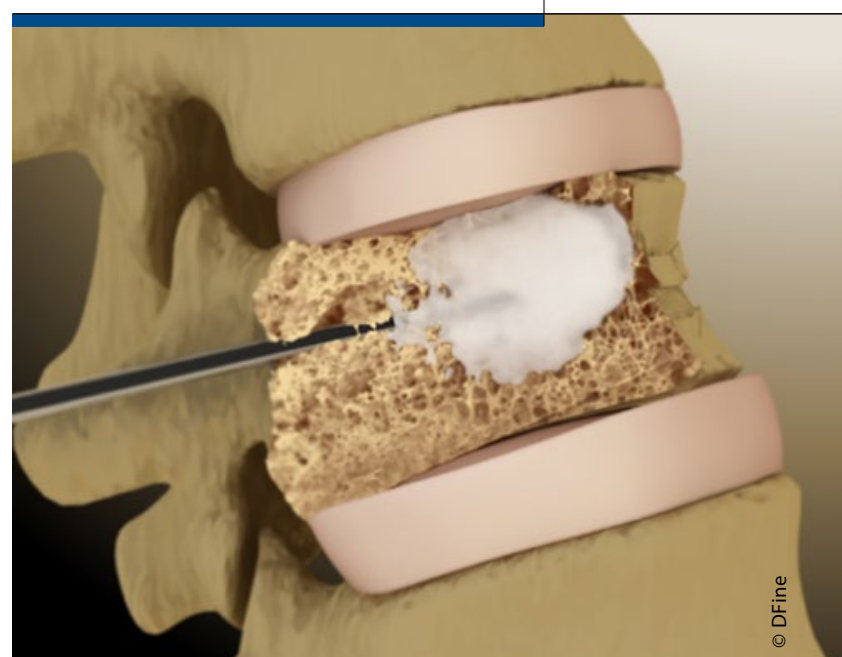

Spezieller Zement wird maschinell in den porösen Wirbelkörper gespritzt und umschließt lückenlos das Knochengewebe, ohne die Mikroarchitektur zu zerstören.

Minimalinvasive Therapie von Wirbelkörperfrakturen

\section{Pluspunkte für die \\ Radiofrequenzkyphoplastie}

Bei der Radiofrequenzkyphoplastie werden Wirbelkörperfrakturen osteoporotischer, onkologischer oder traumatischer Genese durch Einbringen eines speziell entwickelten hochviskösen Knochenzements minimalinvasiv wieder aufgerichtet - knochenschonend, substanzerhaltend und ohne Zuhilfenahme eines Ballons.

$\mathrm{D}$ ie Klinik und Poliklinik für Orthopädie und Unfallchirurgie in Bonn führte eine prospektive Studie zweier Augmentationsverfahren durch [Z Orthop Unfall 2012; 150(1): 56-61; DOI: 10.1055/s-0031-1280122]. Im Zeitraum von Juli 2009 bis September 2010 verglichen die Mediziner die Daten der Ballonkyphoplastie (BKP) mit jenen der Radiofrequenzkyphoplastie (RFK) in zwei Gruppen zu je 114 Patienten. Dabei achteten sie streng auf gleiche Indikation und Ausgangswerte (matched pairs). Als Messparameter für die Wirksamkeit und Sicherheit der beiden Verfahren dienten Schmerzintensität, Wirbelkörperhöhe, Kyphosewinkel, Zementaustritte sowie die Operationsdauer.

Bei der Auswertung ergaben sich zwischen den beiden Behandlungsgruppen signifikante Unterschiede:

_Die Schmerzlinderung war in der RFK-Gruppe deutlich überlegen.

-Die durchschnittliche Operationsdauer reduzierte sich bei der RFK im Vergleich zur BKP etwa um die Hälfte.

_Auch bezüglich der Zementverschleppung schnitt die RFK wesentlich günstiger ab.

_-In der Wirbelkörperhöhe sowie beim Kyphosewinkel stellten die Mediziner in ihrer Studie keine nennenswerten Unterschiede fest.

Krankenkassen erstatten die Kosten für die RKP. Die Patienten profitieren neben der hohen Sicherheit, von einer schnelleren Rehabilitationszeit.

Nach Informationen von DFine Europe

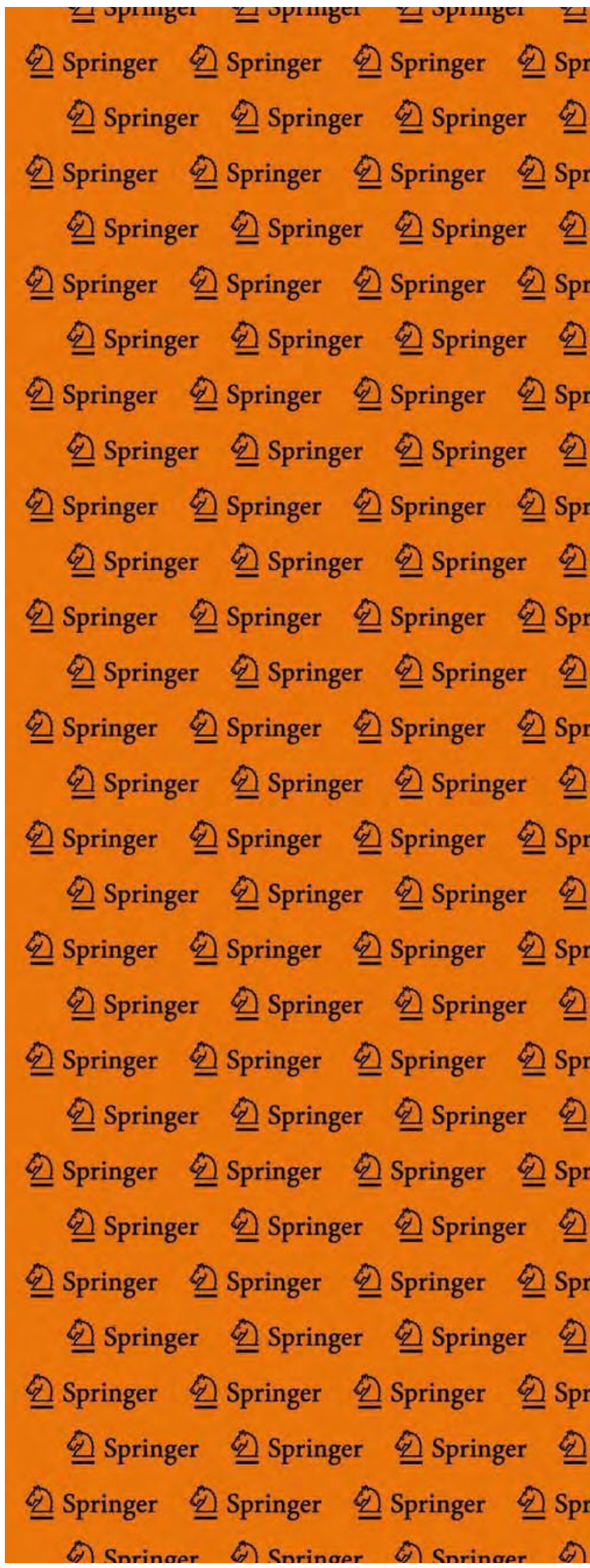
至Springer $\underline{\text { Springer }}$ Springer Springer $\underline{\text { Springer }}$ Springer $\underline{\text { Spr }}$ 旦Springer $\underline{\text { Springer }}$ Springer Springer $\underline{\text { Springer }}$ Springer $\underline{\text { Spr }}$ 을 Springer 을 Springer 을 Springer Springer $\underline{\underline{2} \text { Springer }}$

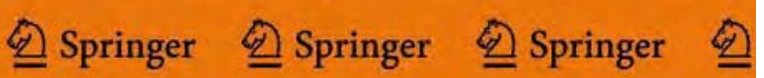
Springer $\underline{\underline{2} \text { Springer }}$ Springer $\underline{\underline{Q}} \mathrm{Spr}$ 을 Springer $\underline{\text { Springer }}$ Springer Springer $\underline{\underline{2} \text { Springer }} \underline{\text { Springer }}$ 을 Springer 슨 Springer Springer $\underline{\text { Springer }} \cong$ Pringer 은 Springer 은 Springer

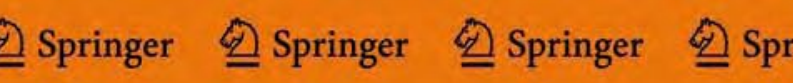

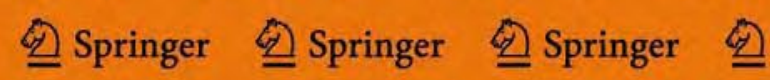

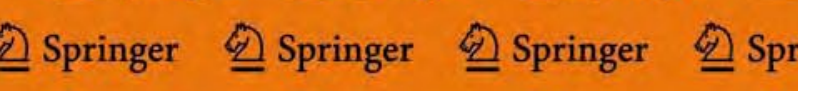
Springer $\underline{\text { Springer }}$ Springer

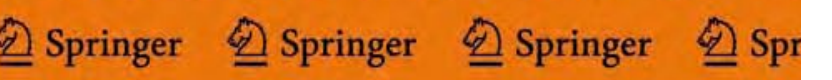
을 Springer Springer Springer Springer Springer $\underline{\text { Springer }}$ Spr 은 Springer $\underline{\text { Springer }}$ Springer

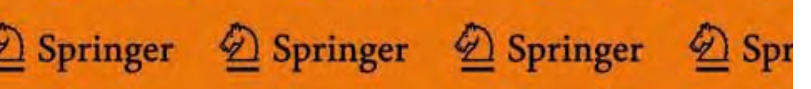
을 Springer $\underline{\text { Springer }}$ Springer 은 Springer $\underline{\text { Springer }}$ Springer $\underline{\text { Spr }}$ Q Springer $\underline{\text { Springer }}$ Springer Springer 2 Springer 2 Springer 2 Spr 